\title{
Magnetic Signatures of Radiation-Driven Double Ablation Fronts
}

\author{
P. T. Campbellø, ${ }^{1,}$ C. A. Walsh $\odot,{ }^{2}$ B. K. Russell $\odot,{ }^{1}$ J. P. Chittenden, ${ }^{2}$ A. Crilly $\odot,{ }^{2}$ \\ G. Fikselø ${ }^{1}$ P. M. Nilson, ${ }^{3}$ A. G. R. Thomas $\odot,{ }^{1}$ K. Krushelnick, ${ }^{1}$ and L. Willingale ${ }^{1}$ \\ ${ }^{1}$ Gérard Mourou Center for Ultrafast Optical Science, University of Michigan, \\ 2200 Bonisteel Boulevard, Ann Arbor, Michigan 48109, USA \\ ${ }^{2}$ Blackett Laboratory, Imperial College, London SW7 2AZ, United Kingdom \\ ${ }^{3}$ Laboratory for Laser Energetics, University of Rochester, 250 East River Road, Rochester, New York 14623, USA
}

(Received 20 April 2020; revised 4 August 2020; accepted 10 August 2020; published 28 September 2020)

In experiments performed with the OMEGA EP laser system, magnetic field generation in double ablation fronts was observed. Proton radiography measured the strength, spatial profile, and temporal dynamics of self-generated magnetic fields as the target material was varied between plastic, aluminum, copper, and gold. Two distinct regions of magnetic field are generated in mid- $Z$ targets—one produced by gradients from electron thermal transport and the second from radiation-driven gradients. Extended magnetohydrodynamic simulations including radiation transport reproduced key aspects of the experiment, including field generation and double ablation front formation.

DOI: 10.1103/PhysRevLett.125.145001

High power laser-matter interactions can create high energy density (HED) conditions with extreme temperatures, densities, and pressures in the laboratory [1]. Strong magnetic fields $(\sim \mathrm{MG})$ can be self-generated in laserproduced plasmas [2-5]. The generation and spatial profile of such magnetic fields can have important consequences for heat transport in inertial confinement fusion (ICF) plasmas [6-11], potentially affecting the uniformity of the radiation drive in hohlraums and impacting the threshold for ignition. Additionally, these self-generated fields enable laboratory investigations of astrophysically relevant plasma phenomena [12], particularly magnetic reconnection [13-18].

At moderate intensities, $I_{L}=10^{14}-10^{15} \mathrm{~W} \mathrm{~cm}^{-2}$, the laser pulse ablates the surface of the dense target. Laser energy is transported beyond the critical density by electron thermal conduction [19]. In low- $Z$ targets, low emissivity means the ablation dynamics are unaffected by radiation transport. Perpendicular temperature and density gradients in the ablated plasma plume generate azimuthal magnetic fields $(\mathcal{O}$ MG) via the Biermann battery mechanism $\left(\partial B / \partial t \propto \nabla T_{e} \times \nabla n_{e}\right)$ [20]. Strong temperature gradients can result in field advection through the Nernst effect $\left(\propto-T_{e}^{3 / 2} \nabla T_{e}\right)[6,8,9,21]$. Counter to the bulk plasma flow out into the corona, the Nernst effect can move fields with the heat flow toward the ablation region. While plasma ions are typically weakly magnetized, self-generated fields can still impact energy flow [9-11].

At higher $Z$, the hot coronal plasma will emit strong $\mathrm{x}$-ray radiation that is absorbed in dense, opaque regions. This establishes a radiation-driven ablation front that is separated from the thermal electron front by a "plateau" of relatively flat density and temperature profiles. As a result, there are two distinct regions with strong gradients that can independently initiate Biermann battery magnetic field generation. Precise details of these double ablation front (DAF) structures, such as the spatial extent and temporal evolution of the plateau region, depend on the target material. An illustration of the ablation dynamics is shown in Fig. 1.

Previous research has focused on using DAF structures to inhibit laser-plasma instabilities and stabilize direct-drive ICF implosions [22-26], or as a platform for laboratory investigations of stellar opacity [27]. Analytic predictions of DAF dynamics are primarily supported using 1D

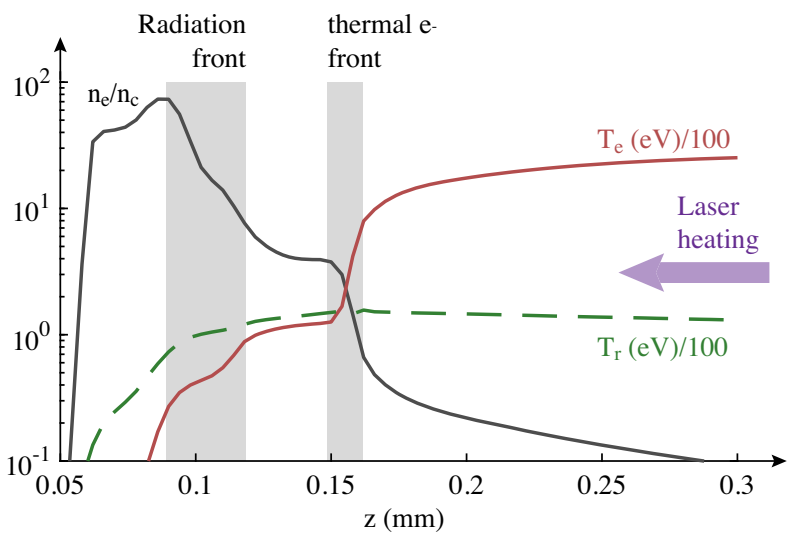

FIG. 1. Results from a 2D radiation-magnetohydrodynamic simulation of a laser pulse interacting with a copper target show evidence of double ablation front formation. Lineouts of the electron density (black line), electron temperature (red line), and radiation temperature (dashed green line) are taken along the laser axis after 750 ps of laser heating. 
simulations [23,24], and consideration of multidimensional effects has been limited to instability stabilization [22]. Thus far, magnetic fields have been neglected.

Measurements of magnetic field generation can validate essential numerical models used to make predictions for HED and ICF research. Experiments with low- $Z$ targets demonstrated that magnetohydrodynamics (MHD) modeling must be extended to include Nernst advection, resistive magnetic diffusion, and cross-field Righi-Leduc heat flow to accurately predict the spatial profile of the magnetic fields [28]. However, computational efforts often center on high $-Z$ materials (typically gold) used in hohlraums for indirect-drive ICF $[10,11]$. Coupling between thermal conduction, radiation transport, and magnetic field generation likely influences energy flow in mid- to high- $Z$ plasmas, as well as during ICF implosions [29].

In this Letter, we use high resolution proton radiography measurements to investigate target material effects on the magnetic fields generated during high power lasersolid interactions. The experimental results coupled with extended radiation-MHD simulations demonstrate that radiation-driven double ablation front structures in mid- $Z$ targets establish two distinct regions of Biermann battery field generation. The magnetic field profile can be used to diagnose temperature and density gradients in this HED system, and illustrate the interplay between heat flow, radiation transport, and field generation.

Experiments were performed with the OMEGA EP laser system at the University of Rochester's Laboratory for Laser Energetics. High power, moderate intensity interactions were driven by a long pulse beam with a $351 \mathrm{~nm}$ wavelength, $1250 \mathrm{~J}$ of energy, and $1 \mathrm{~ns}$ square temporal profile. The beam was focused to an $820-\mu$ m-diameter super-Gaussian spot with a $\sim 30^{\circ}$ angle of incidence to produce a peak intensity of $2 \times 10^{14} \mathrm{~W} \mathrm{~cm}^{-2}$. Thin foil targets were either $50 \mu \mathrm{m}$ thick plastic $(\mathrm{CH}), 25 \mu \mathrm{m}$ copper, $25 \mu \mathrm{m}$ aluminum, or $50 \mu \mathrm{m}$ aluminum coated with either $1 \mu \mathrm{m}$ of copper $(\mathrm{Cu}+\mathrm{Al})$ or gold $(\mathrm{Au}+\mathrm{Al})$.

As shown in Fig. 2, protons accelerated by target normal sheath acceleration [30] were used to image the magnetic fields in a point-projection geometry. An infrared (IR) laser with $300 \mathrm{~J}$ in a $0.7 \mathrm{ps}$ pulse was focused to intensities

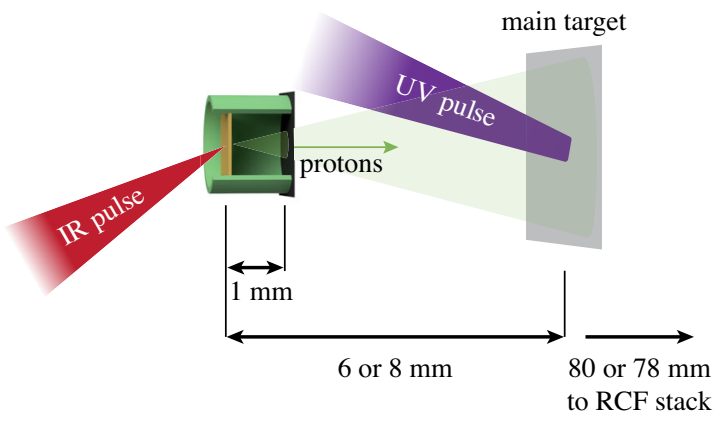

FIG. 2. Schematic of the OMEGA EP experiment. exceeding $10^{20} \mathrm{~W} \mathrm{~cm}^{-2}$ onto $1 \times 1 \mathrm{~mm}^{2}$ foils, typically $50-\mu \mathrm{m}$-thick copper. The proton source foil was protected from preheat using a target assembly similar to that described in Ref. [28] and produced a quasi-Maxwellian energy spectrum with maximum energies exceeding $60 \mathrm{MeV}$. The deflected proton beam was detected on stacks of radiochromic film (RCF). Quantitative measurements of the path-integrated magnetic field can be retrieved from the relative transverse deflections of protons in the beam $[31,32]$. The relative timing between laser pulses could be adjusted with $\pm 20 \mathrm{ps}$ error to measure the temporal dynamics of the evolving magnetic fields.

Experimental results were compared to two-dimensional (2D), cylindrically symmetric simulations performed using the extended-MHD code, GORGON [29,33-35]. GORGON includes magnetic transport from bulk plasma flow, Nernst, cross-gradient Nernst, resistive diffusion, and Biermann battery generation [35]. The thermal transport is anisotropic and includes Righi-Leduc heat flow. Multigroup nondiffusive radiation transport is implemented using a $P_{1 / 3}$ automatic flux-limiting method. The Frankfurt equation of state [36] is used along with a Thomas-Fermi ionization model. The laser is treated using a ray-trace method with inverse-bremsstrahlung heating of the electron population.

Figure 3 shows experimental and computational observations of the temporal evolution of magnetic signatures of double ablation fronts on copper foils. While significant blurring due to scattering in the solid copper target [Fig. 3(a)] limits the imaging resolution, two distinct rings of proton accumulation, evidence of the DAF structure, can be resolved after 750 ps. The layered $\mathrm{Cu}+\mathrm{Al}$ targets [Fig. 3(b)] enabled higher resolution proton images. The key features are reproduced with improved clarity due to reduced proton scattering through the bulk aluminum.

Corresponding 2D magnetic field profiles from a GORGON simulation of a copper target interaction are shown in Fig. 3(c). Two radially separated regions of MG-level fields have evolved by $0.5 \mathrm{~ns}$ and continue to grow as time progresses. Concentric bands of field generation are the result of steep temperature gradients at each of the DAF heat fronts (see Fig. 1). Near the edge of the ablated plume, the temperature gradients are primarily directed radially and persist further from the target surface. Additionally, x-ray radiation emitted from the corona can preheat the target surface outside the laser focal area. The interaction of the plume with the preexpanding surface redirects the bulk plasma flow, pushing the magnetic field away from the target surface.

In Figs. 3(d) and 3(e), lineouts from the experimental proton images are compared to synthetic radiographs produced using particle tracking through the simulated fields. Note that the experimental results exhibit an elliptical shape due to the laser angle of incidence. To achieve the best comparison with the normal incidence $2 \mathrm{D}$ simulation, the experimental lineouts are taken along the 

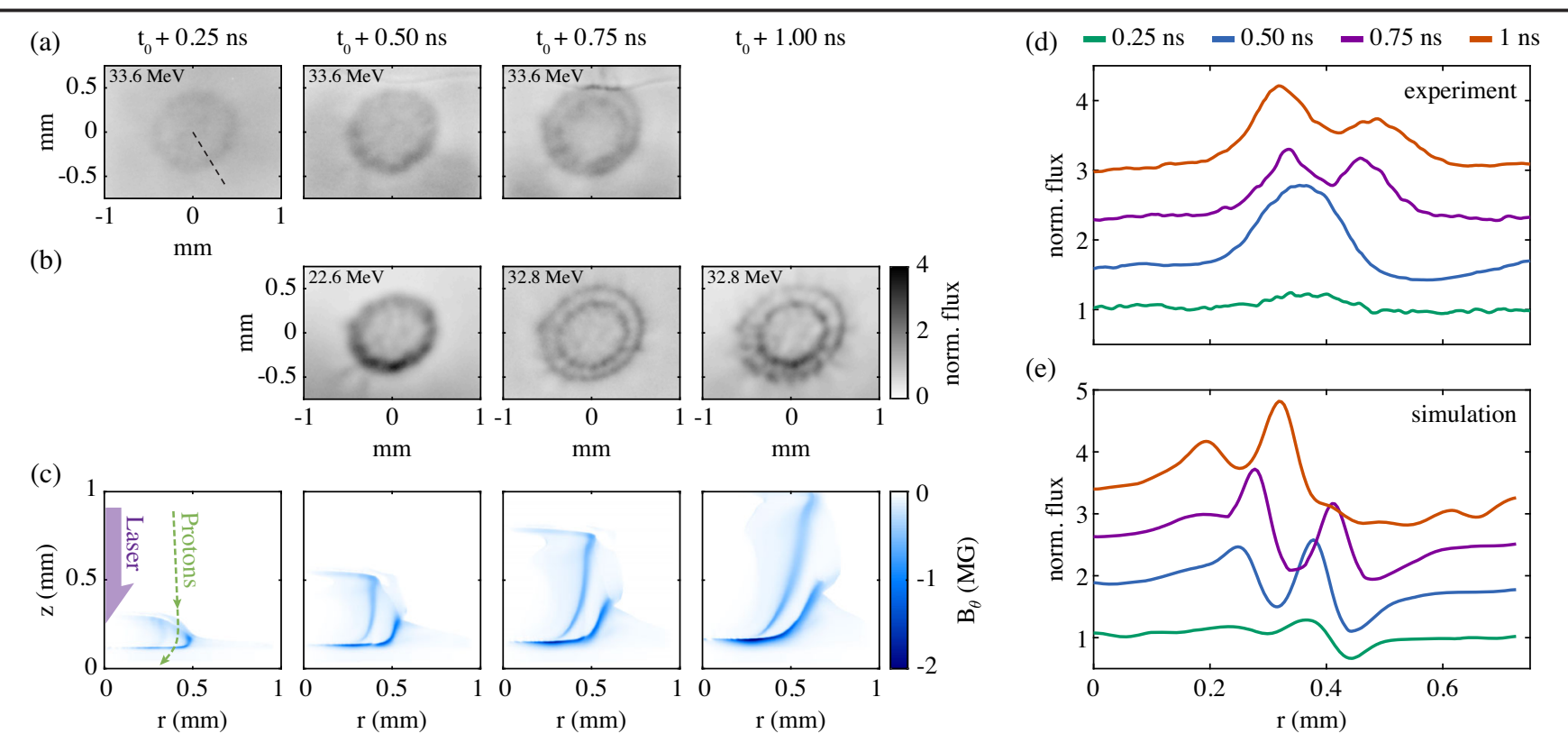

FIG. 3. Experimental proton images using $\mathrm{Cu}$ (a) and $\mathrm{Cu}+\mathrm{Al}$ (b) targets show the formation of two concentric rings of proton accumulation. Magnetic field profiles from corresponding times in the $2 \mathrm{D}(r-z)$ cylindrically symmetric simulation (c) reveal the evolution of two, radially distinct magnetic field regions. Similar trends can be observed when comparing lineouts through the experimental proton images (d) and synthetic images generated using the simulation data (e) (lineouts are offset vertically for clarity, with later times in higher positions). Experimental lineouts were taken along the dashed line shown in (a) from the $\mathrm{Cu}+\mathrm{Al}$ results, except at $0.25 \mathrm{~ns}$. Synthetic images were produced with $33.6 \mathrm{MeV}$ protons for 0.25 through $0.75 \mathrm{~ns}$ and $32.8 \mathrm{MeV}$ for $1 \mathrm{~ns}$.

minor axis [dashed line shown in Fig. 3(a)]. The simulated radiographs have been blurred using a $50 \mu \mathrm{m}$ FWHM Gaussian to approximate the effect of scattering.

Proton image features are due to both the radial position and path-integrated strength of the magnetic fields [32]. Similar trends are observed in the experimental and simulation results. The synthetic proton images reproduce the experimental observation of two rings of proton accumulation attributed to the double magnetic field structure. However, the double field appears earlier in the simulation, and features in the synthetic proton images occur at smaller radii. These discrepancies are likely due to nonequilibrium effects on the radiation transport and kinetic effects, such as nonlocal transport, that are not included in the model. For example, recent work using kinetic simulations has demonstrated that MHD models overestimate the rate of Biermann battery field generation in plasmas with long electron mean free paths [37,38]. In this case, artificially enhanced field strengths would result in overfocusing of the protons to produce features at narrower radii.

At later times in Fig. 3(c), the internal band of field extends far from the target surface into the hot corona. The combination of low density and high temperature in this region yields relatively long Coulomb mean free paths (tens of micrometers), meaning the rate of field generation could be exaggerated. Estimates based on Ref. [37] suggest a suppression of the Biermann battery generation rate by factors ranging from $\sim 0.2$ to 0.5 across the plume (additional details about this calculation, including comparisons with experimental results, can be found in the Supplemental Material [39]). These experimental results could be used to verify kinetic predictions of Biermann battery suppression.

Further insight into the connection between DAFs and magnetic field structures can be gained by comparing target materials. Figure 4 shows 2D magnetic field and electron density profiles from GORGON simulations of $\mathrm{CH}$ and $\mathrm{Cu}$ target interactions at $t_{0}+0.75 \mathrm{~ns}$. In general, the simulations of the $\mathrm{CH}$ target agree with previous work $[28,40]$. A single band of azimuthal magnetic field with $\mathcal{O}$ (MG) peak strength is generated in the strong temperature and density gradients near the edge of the laser focal spot.

Radial lineouts through the magnetic field, electron temperature, and Nernst velocity for each material are plotted in Figs. 4(c) and 4(d). The lineouts show the spatial relationship between the strong temperature gradients and the magnetic field generation. The two distinct radial regions of field generation are evident in the copper simulation. Between the two fields is a plateau region of relatively low temperature gradient, a signature of DAF structures. As a result, the Nernst effect $\left(\mathbf{v}_{N} \propto-T_{e}^{3 / 2} \nabla T_{e}\right)$ is suppressed and the inner field is not advected toward the outer field. This allows magnetic field to remain in the hotter, low density plasma, which is more readily magnetized.

Overlaid on Figs. 4(a) and 4(b) are contours of electron temperature and selected effective ionization states $\left(Z_{\text {eff }}\right)$. 


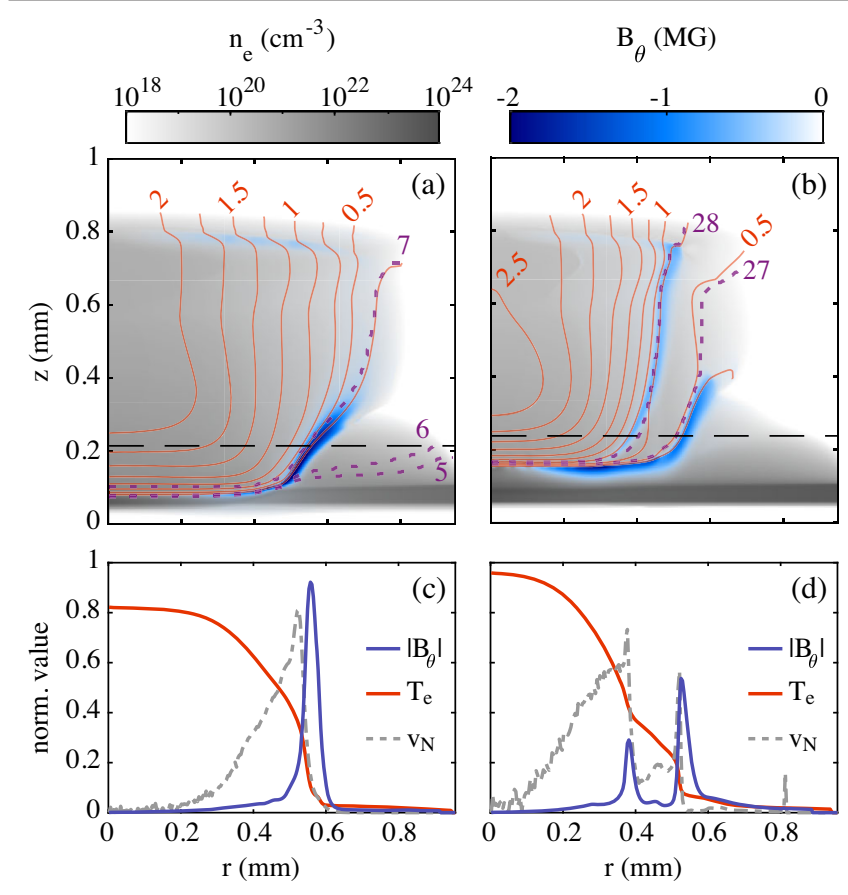

FIG. 4. 2D simulation results for $\mathrm{CH}$ (a) and $\mathrm{Cu}$ (b) plasmas at a $t_{0}+0.75 \mathrm{~ns}$. Magnetic field profiles (blue) are overlaid on the electron density (gray). $T_{e}$ contours (in units of $\mathrm{keV}$ ) are shown in red, and select contours of $Z_{\text {eff }}$ are plotted with dashed purple lines. Panels (c) and (d) show radial profiles [along the dashed lines indicated in (a) and (b)] of the magnetic field (blue line), $T_{e}$ (red line), and Nernst velocity ( $\mathbf{v}_{N}$, dashed gray line). Magnetic field lineouts are normalized by $2 \mathrm{MG}, T_{e}$ by $2.25 \mathrm{keV}$, and $\mathbf{v}_{N}$ by $1.5 \times 10^{6} \mathrm{~m} / \mathrm{s}$.

The $\mathrm{CH}$ target exhibits a nearly uniform, fully ionized state within the plasma plume. For copper, x-ray radiation emitted from the hottest regions drives additional heating and initiates ionization from a He-like to H-like state in the plasma near the edge of the plume.

This suggests ionization is key to DAF formation in regions where temperature gradients are perpendicular to density gradients (critical for Biermann battery magnetic field generation). Ionization processes enable DAF structures to develop by sharply increasing the opacity as the temperature drops near the edges of the plume, even without a strong antiparallel density ramp as found along the laser axis (see Fig. 1).

The top row of Fig. 5 compares experimental proton images taken at $0.75 \mathrm{~ns}$ for different target materials: $\mathrm{CH}$, $\mathrm{Al}, \mathrm{Cu}+\mathrm{Al}$, and $\mathrm{Au}+\mathrm{Al}$. In all cases, we observe a strong ring pattern of proton accumulation. The $\mathrm{CH}$ results closely resemble those published by Gao et al. [28], with a pronounced dark ring pattern associated with azimuthal fields surrounding the laser focal spot. The primary difference between the different materials is the emergence of a second prominent concentric ring of proton accumulation for mid- $Z$ targets, with the ring separation increasing between the $\mathrm{Al}$ and $\mathrm{Cu}+\mathrm{Al}$ targets. The highest $Z$ target, $\mathrm{Au}$, results in a single ring at smaller radius.
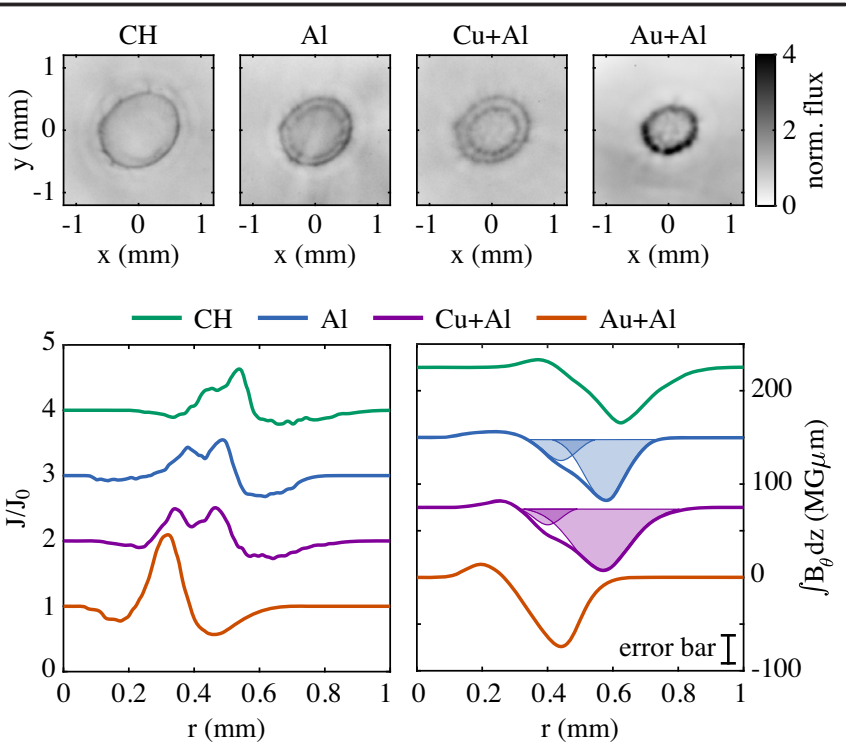

FIG. 5. Proton images (top row) and field reconstruction analysis (bottom row) are compared for the different target materials at $t_{0}+0.75 \mathrm{~ns}$. The proton energy is $37.3 \mathrm{MeV}$ for $\mathrm{CH}, \mathrm{Al}$, and $\mathrm{Cu}+\mathrm{Al}$, and $30.7 \mathrm{MeV}$ for $\mathrm{Au}+\mathrm{Al}$. Bottom left: the radial lineouts $(J)$, normalized by the mean inferred reference profile $\left(J_{0}\right)$. Bottom right: the resulting reconstructed field profiles. For $\mathrm{Al}$ and $\mathrm{Cu}+\mathrm{Al}$; the results of double-Gaussian fitting are shown with shaded regions.

Although elongated due to the angle of incidence, the ring features are approximately azimuthally symmetric. A 1D polar-coordinates field reconstruction technique, described in the Supplemental Material [39], was developed to extract quantitative path-integrated magnetic field information based on Ref. [32]. For each material, radial lineouts of the proton fluence taken along the minor axis were inverted to calculate $\int B_{\theta} d z$.

The mean normalized proton fluence, $J / J_{0}$, and inverted path-integrated magnetic field for each material are shown in the lower left- and right-hand plots of Fig. 5, respectively. To further characterize the uncertainty of this method, successive layers of RCF were analyzed (note that the relative time-of-flight differences are small compared to the interaction timescale). Overall, uncertainties in determining $J_{0}$ lead to large errors in absolute field strength $(\sim \pm 15 \mathrm{MG} \mu \mathrm{m})$. Additionally, the accuracy will also be impacted by blurring due to small-angle proton scattering, and potentially by enhanced proton stopping in the heated target [41,42]. Finally, the deflections are assumed to be due to magnetic fields only. GORGON simulations confirm electric fields are relatively weak.

Changing the material from $\mathrm{CH}$ to $\mathrm{Al}$ or $\mathrm{Cu}$, an interior field structure emerges corresponding to the double ring feature observed in the images. This is evidence of the formation of the second ablation front region. Fundamentally, DAF dynamics depend on a balance of radiation energy flux and opacity [23,24]. While the 
radiation flux is too low in $\mathrm{CH}$, a combination of more efficient emission and higher opacities in mid- $Z$ targets initiates DAF formation.

The plateau width scales as the product of radiation mean free path and energy flux [23]. Figure 5 shows doubleGaussian fits to the reconstructed fields (shaded area). The field peak separation $(\Delta r)$ is analogous to the plateau width, and increases from $0.14 \mathrm{~mm}$ in $\mathrm{Al}$ to $0.17 \mathrm{~mm}$ in $\mathrm{Cu}+\mathrm{Al}$. The enhanced radiation energy flux from $\mathrm{Cu}$ overcomes the shorter mean free paths expected at higher $Z$. However, for even higher $Z$, the balance could shift as opacity increases to reduce the mean free path, and therefore the front separation, despite more efficient radiation emission.

A single field feature is observed using the $\mathrm{Au}+\mathrm{Al}$ target. The slow hydrodynamic evolution due to the heavy ions mean the peak field occurs at the smallest radius. Signatures of DAFs may potentially develop at later times, but would likely be below the measurement resolution due to short radiation mean free paths and enhanced proton scattering in Au.

In summary, magnetic field generation has been observed in radiation-driven double ablation front structures. Qualitative and reasonable quantitative agreement was found between the experimental results and radiation extended-MHD simulations. The results illuminate how ionization dynamics contribute to DAF formation and how suppression of the Nernst effect within the temperature plateau region influences the magnetic field profile.

DAF formation is anticipated in direct-drive ICF experiments using mid- $Z$ or doped ablators $[22,25]$. The associated magnetic field generation observed here potentially affects heat flow and fast electron propagation during the implosion [38]. In addition, a detailed understanding of the interplay of heat transport, magnetic fields, and radiation physics is crucial for accurate modeling of hohlraum dynamics.

This experimental platform is much simpler to diagnose than an ICF implosion or hohlraum. The results can be used to verify aspects of extended-MHD and radiation transport calculations, as well as the importance of kinetic effects like suppression of Biermann battery generation due to nonlocal transport. Leveraging this platform to advance and refine computational models could provide an essential tool for accurately simulating hohlraum physics and for future astrophysically relevant plasma studies.

This material is based upon work supported by the Department of Energy, National Nuclear Security Administration under Awards No. DE-NA0003606 and No. DE-NA0003764. P. T.C. is supported by the U.S. Department of Energy Fusion Energy Sciences Postdoctoral Research Program administered by the Oak Ridge Institute for Science and Education (ORISE) for the DOE. ORISE is managed by Oak Ridge Associated Universities (ORAU) under DOE Contract No. DESC0014664. All opinions expressed in this paper are the authors' and do not necessarily reflect the policies and views of DOE, ORAU, or ORISE. B. K. R. acknowledges support from NSF Grant No. 175142. Simulation results reported in this Letter were obtained using the Imperial College High Performance Computer Cx1.

*campbpt@umich.edu

[1] R. P. Drake, High Energy Density Physics: Fundamentals, Inertial Fusion and Experimental Astrophysics, 2nd ed. (Springer International Publishing, Berlin, 2018).

[2] J. A. Stamper, K. Papadopoulos, R. N. Sudan, S. O. Dean, E. A. McLean, and J. M. Dawson, Phys. Rev. Lett. 26, 1012 (1971).

[3] J. A. Stamper and B. H. Ripin, Phys. Rev. Lett. 34, 138 (1975).

[4] A. Raven, O. Willi, and P. T. Rumsby, Phys. Rev. Lett. 41, 554 (1978).

[5] M. G. Haines, Can. J. Phys. 64, 912 (1986).

[6] T. H. Kho and M. G. Haines, Phys. Rev. Lett. 55, 825 (1985).

[7] D. H. Froula, J. S. Ross, B. B. Pollock, P. Davis, A. N. James, L. Divol, M. J. Edwards, A. A. Offenberger, D. Price, R. P. J. Town et al., Phys. Rev. Lett. 98, 135001 (2007).

[8] C. P. Ridgers, R. J. Kingham, and A. G. R. Thomas, Phys. Rev. Lett. 100, 075003 (2008).

[9] L. Willingale, A. G. R. Thomas, P. M. Nilson, M. C. Kaluza, S. Bandyopadhyay, A. E. Dangor, R. G. Evans, P. Fernandes, M. G. Haines, C. Kamperidis et al., Phys. Rev. Lett. 105, 095001 (2010).

[10] A. S. Joglekar, C. P. Ridgers, R. J. Kingham, and A. G. R. Thomas, Phys. Rev. E 93, 043206 (2016).

[11] W. A. Farmer, J. M. Koning, D. J. Strozzi, D. E. Hinkel, L. F. Berzak Hopkins, O.S. Jones, and M. D. Rosen, Phys. Plasmas 24, 052703 (2017).

[12] B. A. Remington, R. P. Drake, and D. D. Ryutov, Rev. Mod. Phys. 78, 755 (2006).

[13] P. M. Nilson, L. Willingale, M. C. Kaluza, C. Kamperidis, S. Minardi, M. S. Wei, P. Fernandes, M. Notley, S. Bandyopadhyay, M. Sherlock et al., Phys. Rev. Lett. 97, 255001 (2006).

[14] C. K. Li, F. H. Séguin, J. A. Frenje, J. R. Rygg, R. D. Petrasso, R. P. J. Town, O. L. Landen, J. P. Knauer, and V. A. Smalyuk, Phys. Rev. Lett. 99, 055001 (2007).

[15] L. Willingale, P. M. Nilson, M. C. Kaluza, A. E. Dangor, R. G. Evans, P. Fernandes, M. G. Haines, C. Kamperidis, R. J. Kingham, C. P. Ridgers et al., Phys. Plasmas 17, 043104 (2010).

[16] J. Zhong, Y. Li, X. Wang, J. Wang, Q. Dong, C. Xiao, S. Wang, X. Liu, L. Zhang, L. An et al., Nat. Phys. 6, 984 (2010).

[17] W. Fox, A. Bhattacharjee, and K. Germaschewski, Phys. Rev. Lett. 106, 215003 (2011).

[18] Q.-L. Dong, S.-J. Wang, Q.-M. Lu, C. Huang, D.-W. Yuan, X. Liu, X.-X. Lin, Y.-T. Li, H.-G. Wei, J.-Y. Zhong et al., Phys. Rev. Lett. 108, 215001 (2012).

[19] J. Lindl, Phys. Plasmas 2, 3933 (1995).

[20] L. Biermann and A. Schlüter, Phys. Rev. 82, 863 (1951).

[21] A. Nishiguchi, T. Yabe, M. G. Haines, M. Psimopoulos, and H. Takewaki, Phys. Rev. Lett. 53, 262 (1984). 
[22] S. Fujioka, A. Sunahara, K. Nishihara, N. Ohnishi, T. Johzaki, H. Shiraga, K. Shigemori, M. Nakai, T. Ikegawa, M. Murakami et al., Phys. Rev. Lett. 92, 195001 (2004).

[23] J. Sanz, R. Betti, V. A. Smalyuk, M. Olazabal-Loume, V. Drean, V. Tikhonchuk, X. Ribeyre, and J. Feugeas, Phys. Plasmas 16, 082704 (2009).

[24] V. Drean, M. Olazabal-Loum, J. Sanz, and V. T. Tikhonchuk, Phys. Plasmas 17, 122701 (2010).

[25] V. A. Smalyuk, R. Betti, J. A. Delettrez, V. Y. Glebov, D. D. Meyerhofer, P. B. Radha, S. P. Regan, T. C. Sangster, J. Sanz, W. Seka et al., Phys. Rev. Lett. 104, 165002 (2010).

[26] M. Lafon, R. Betti, K. S. Anderson, T. J. B. Collins, R. Epstein, P. W. McKenty, J. F. Myatt, A. Shvydky, and S. Skupsky, Phys. Plasmas 22, 032703 (2015).

[27] A. Colaïtis, J.-E. Ducret, M. Le Pennec, X. Ribeyre, and S. Turck-Chiéze, Phys. Plasmas 25, 072707 (2018).

[28] L. Gao, P. M. Nilson, I. V. Igumenshchev, M. G. Haines, D. H. Froula, R. Betti, and D. D. Meyerhofer, Phys. Rev. Lett. 114, 215003 (2015).

[29] C. A. Walsh, J. P. Chittenden, K. McGlinchey, N. P. L. Niasse, and B. D. Appelbe, Phys. Rev. Lett. 118, 155001 (2017).

[30] A. Maksimchuk, S. Gu, K. Flippo, D. Umstadter, and V. Y. Bychenkov, Phys. Rev. Lett. 84, 4108 (2000); R. A. Snavely, M. H. Key, S. P. Hatchett, T. E. Cowan, M. Roth, T. W. Phillips, M. A. Stoyer, E. A. Henry, T. C. Sangster, M. S. Singh, S. C. Wilks, A. MacKinnon, A. Offenberger, D. M. Pennington, K. Yasuike, A. B. Langdon, B. F. Lasinski, J. Johnson, M. D. Perry, and E. M. Campbell, Phys. Rev. Lett. 85, 2945 (2000); E. L. Clark, K. Krushelnick, J. R. Davies, M. Zepf, M. Tatarakis, F. N. Beg, A. Machacek, P. A. Norreys, M. I. K. Santala, I. Watts, and A. E. Dangor, Phys. Rev. Lett. 84, 670 (2000); S. P. Hatchett, C. G. Brown, T. E. Cowan, E. A. Henry, J. S. Johnson, M. H. Key, J. A. Koch, A. B. Langdon, B. F. Lasinski, R. W. Lee et al., Phys. Plasmas 7, 2076 (2000).
[31] M. Borghesi, A. Schiavi, D. H. Campbell, M. G. Haines, O. Willi, A. J. MacKinnon, L. A. Gizzi, M. Galimberti, R. J. Clarke, and H. Ruhl, Plasma Phys. Controlled Fusion 43, A267 (2001).

[32] N. L. Kugland, D. D. Ryutov, C. Plechaty, J. S. Ross, and H.-S. Park, Rev. Sci. Instrum. 83, 101301 (2012).

[33] J. P. Chittenden, S. V. Lebedev, C. A. Jennings, S. N. Bland, and A. Ciardi, Plasma Phys. Controlled Fusion 46, B457 (2004).

[34] A. Ciardi, S. V. Lebedev, A. Frank, E. G. Blackman, J. P. Chittenden, C. J. Jennings, D. J. Ampleford, S. N. Bland, S. C. Bott, J. Rapley et al., Phys. Plasmas 14, 056501 (2007).

[35] C. A. Walsh, J. P. Chittenden, D. W. Hill, and C. Ridgers, Phys. Plasmas 27, 022103 (2020).

[36] S. Faik, A. Tauschwitz, and I. Iosilevskiy, Comput. Phys. Commun. 227, 117 (2018).

[37] M. Sherlock and J. J. Bissell, Phys. Rev. Lett. 124, 055001 (2020).

[38] D. W. Hill and R. J. Kingham, Phys. Rev. E 98, 021201(R) (2018).

[39] See Supplemental Material at http://link.aps.org/ supplemental/10.1103/PhysRevLett.125.145001 for additional details about kinetic suppression of Biermann battery generation and the $1 \mathrm{D}$ polar-coordinates field reconstruction method.

[40] L. Lancia, B. Albertazzi, C. Boniface, A. Grisollet, R. Riquier, F. Chaland, K.-C. Le Thanh, P. Mellor, P. Antici, S. Buffechoux et al., Phys. Rev. Lett. 113, 235001 (2014).

[41] A. B. Zylstra, J. A. Frenje, P. E. Grabowski, C. K. Li, G. W. Collins, P. Fitzsimmons, S. Glenzer, F. Graziani, S. B. Hansen, S. X. Hu et al., Phys. Rev. Lett. 114, 215002 (2015).

[42] M. J.-E. Manuel, A. B. Zylstra, H. G. Rinderknecht, D. T. Casey, M. J. Rosenberg, N. Sinenian, C. K. Li, J. A. Frenje, F. H. Séguin, and R. D. Petrasso, Rev. Sci. Instrum. 83, 063506 (2012). 